\title{
Table Salt is An Alternative to Silver Nitrate Cautery for the Treatment of Umbilical Granuloma: A Prospective Randomized Controlled Trial.
}

\author{
Tulin Oztas (Corresponding author) \\ Department of Pediatric Surgery, University of Health Sciences Gazi Yasargil Training and Research \\ Hospital, Diyarbakir, Turkey \\ E-mail: tulinoztas@ hotmail.com \\ Ahmet Dursun \\ Department of Pediatric Surgery, University of Health Sciences Gazi Yasargil Training and Research \\ Hospital, Diyarbakir, Turkey \\ Salim Bilici \\ Department of Pediatric Surgery, University of Health Sciences Gazi Yasargil Training and Research \\ Hospital, Diyarbakir, Turkey
}

\begin{abstract}
The aim of this prospective study explore a cheap and readily available alternative to silver nitrate cautery, with far-reaching implications for many populations with limited access to medical care. Seventy four patients were enrolled in this prospective randomized controlled study. Patients were divided in two groups of 37 patients each. A stick applicator of $75 \%$ silver nitrate was applied in first group by the pediatric surgeon in the outpatient clinic. The patients in the second group was applied a small amount of table salt granules twice a day for 3 days by the family members at home. In the first week after treatment, the same treatment was applied for the second time to the patients who presence of umbilical exudate in both groups. Response to treatment was evaluated in the second week. There were no statistically significant differences between the two groups in the first week and second week after treatment in regard to presence of umbilical exudate and complication $(\mathrm{P}>0,05)$. Table salt is a safe alternative to silver nitrate cautery in the treatment of umbilical granuloma.
\end{abstract}

Key words: Silver nitrate; table salt; umbilical granuloma

\section{Special Issue of Health Sciences}

DOI: $10.7176 /$ JSTR/6-03-33

\section{Introduction}

Umbilical granuloma (UG) is the most common benign umbilical pathology in infants (Badebarin et al., 2018; Dhungel et al., 2008). The emergent granulation tissue after the separation of the umbilical cord may turn into a granuloma due to an insufficient epithelization of the umbilical ring (Annapurna et al., 2015; Hossain et al., 2010; Fiaz et al., 2017; Saleh 2016; Pomeranz A 2004). Cauterization with silver nitrate or copper sulfate, electrocauterization, cryotherapy, double ligature, surgical excision, topical administration of table salt, antibiotics, steroids and the application of absorbable hemostatic material are the treatment options for UG (Fiaz et al., 2017; Saleh 2016; Pomeranz A 2004; Nagar 2001; Ogowa 2018). Electrocauterization and cryotherapy are performed by experienced health care providers at wellequipped health care centers. These treatments are costly and it has been reported that a type of skin discoloration may be seen after their application (Hossain et al., 2010; Sheth et al., 1990) . Another treatment method is double ligature which can be applied to only pedunculated UG (Lotan et al., 2002). Copper sulfate is not easily available, high costs and needs to be applied at the hospital. Furthermore, burns in the skin have been reported after this treatment modality (Dhungel et al., 2008; Annapurna et al.,2015; Hossain et al., 2010; Fiaz et al., 2017). Although local steroid treatment is recommended for UG treatment, it is not recommended for routine use in the treatment of UG because of their local and systemic side effects (Brodsgaard et al., 2015; Aydın et al., 2015). Surgical excision is another invasive 
method used for the treatment when a response cannot be obtained with other methods. This method has high cost and the need to administer general anesthesia to the patient (Hossain et al.,2010). The current published literature sorely lacks medical protocols that are useful and applicable in resource poor settings. The aim of this prospective study explores a cheap and readily available alternative to silver nitrate cautery, with far-reaching implications for many populations with limited access to medical care.

\section{Material and Methods}

Seventy four patients scheduled for treatment of UG in our pediatric surgery outpatient clinic between 01 May 2018 and 31 December 2018 were enrolled in this prospective randomized controlled study. Patients were divided in two groups of 37 patients each. A stick applicator of $75 \%$ silver nitrate was applied in first group by the pediatric surgeon in the outpatient clinic (Group I) The patients in the second group was applied a small amount of table salt granules twice a day for 3 days by the family members at home (Group II). Simple randomization was performed in patient selection. The study protocol was approved by the Clinical Studies Ethics Committee of Health Sciences University may 2018 (decision number 81). Written consent was obtained from the parents of each patients. Gender, gestational age, age at diagnosis, birth weight and umbilical seperation time documented in these patients. Patients were not included if they were older than 5 weeks of age, infection in the umbilicus, prior treatment of UG . The patients in the first group, the umbilical area was cleaned with an antiseptic solution (\%10 povidon iodine solution) and it was left to dry. After drying, a stick applicator of $75 \%$ silver nitrate was applied to the granuloma by the pediatric surgeon in the outpatient clinic.

The patients in the second group umbilical region was cleaned with warm water in the table salt group. Then, a small amount of table salt granules was applied to the granuloma and the region was covered with sterile gauze. The region was uncovered 30 minutes later and cleaned with warm water again before finalizing the treatment. After this procedure was explained to the parent of the patient, it was applied twice a day for 3 days by the family members at home. In order to evaluation umbilical excudate and complication, patients were invited to the hospital in the first and second week after treatment. Presence of umbilical exudate, discoloration of the periumbilical skin, burn of the periumbilical skin of all patients were documented at each visit. After first week treatment, the same treatment was applied for the second time to the patients who continued umbilical exudate in both groups. The patients were contacted four weeks after the treatment via telephone by two independent researchers (one being a nurse who was blind to this study). The patients were asked if they had any complaints about the therapy, if burn or skin discoloration took place and if relapses occured and the answers were documented.

Recovery was defined as absence of umbilical exudate or granuloma. Treatment failure was defined, presence of umbilical exudate or decreased amount of umbilical exudate, presence of granuloma after two weeks treatment.

\section{Statistical Analysis}

Data obtained in the study were analyzed statistically using SPSS (Statistical Package for Social Sciences) version 22.0 software program for Windows. Categorical variables were presented as number (n) and percentage (\%). The Kolmogorov-Smirnov test was used to examine the normality distribution of continuous data. Normally distributed numerical variables were shown as mean plus/minus standard deviation. Normally distributed numeric variables were compared using the Student's T-test. To determine differences between group of silver nitrate and common salt in respect of categorical variables, Chi-square test and Mann-Whitney $U$ test were performed. A value of $\mathrm{p}<0.05$ was considered as statistically significant.

\section{Results}

Seventy four patients aged 5-35 days were enrolled in this study. Of the 74 patients, were 40 men and 34 were women. The patients were divided into two groups according to the aforementioned criteria. There were no statistically signifcant differences between the groups (Table 1).

There were no statistically signifcant differences between the two groups in the first week after treatment considering presence of umbilical exudate $(\mathrm{p}=1.0)$ In the two groups, 7 (23.3\%) patients were presence of umbilical exudate. The same treatment was applied for the second time to this patients. There were no complications in the table salt group after the treatment, however, a first degree burn at the periumbilical area developed in one patient $(2.7 \%)$ in the silver nitrate group. There were no statistically significant differences between the two groups in the first week after treatment in regard to complication $(\mathrm{p}=1.0)($ Table 2). 
Table 1. The demographic characteristics of the patients silver nitrate and common salt group.

\begin{tabular}{llll}
\hline & Group I (n=37) & Group II (n=37) & P \\
\hline Gender & $21(56.8)$ & $19(51.3)$ & \\
Male ,n(\%) & $16(43.2)$ & $18(48.7)$ & 0.64 \\
Female ,n(\%) & $38.9(38-40)$ & $38.8(38-40)$ & 0.59 \\
Gestasyonel age & $17.4(5-35)$ & $17.2(7-35)$ & 0.97 \\
Age at diagnosis (days),median (range) & $3238(2100-4000)$ & $3297(2500-4500)$ & 0.54 \\
$\begin{array}{l}\text { Weight at birth (g),median ((range) } \\
\text { Umbilical cord seperation time (days) }\end{array}$ & & & \\
median range & $7.6(4-15)$ & $7.13(4-17)$ & 0.43 \\
\hline
\end{tabular}

Table 2. Results in the first week after theray

\begin{tabular}{lcccc}
\hline & & Group I $(\mathrm{n}=37)$ & GroupII(n=37) & P \\
\hline Absence of umbilical exudate & $\mathrm{n}(\%)$ & $30(\% 81.08)$ & $30(\% 81.08)$ & 1.0 \\
Resence of umbilical exudate & $\mathrm{n}(\%)$ & $7(\% 23.3)$ & $7(\% 23.3)$ & 1.0 \\
Complication & & $1(\% 2.7)$ & - & 1.0 \\
Burn & $\mathrm{n}(\%)$ & - & - & \\
Skin discoloration & $\mathrm{n}(\%)$ & &
\end{tabular}

There were no statistically signifcant differences between the two groups in the second week after treatment regarding recovery $(\mathrm{p}=0.49)$. Recovery rates were $94,5 \%(\mathrm{n}=35)$ and $100 \%(\mathrm{n}=37)$ in silver nitrate group and the table salt group, respectively. In the silver nitrate group, $5.4 \%(\mathrm{n}=2)$ patients did not respond to the treatment at the second week. There were no complications in the table salt group, but, skin discoloration at the periumbilical area developed in two patients (5.4\%) in the silver nitrate group. There were no statistically significant differences between the two groups in the second week after treatment considering complication $(\mathrm{p}=0.49)$ ( Table 3$)$.

In the telephone interviews done four week after study were no recurrence in both groups.

Table 3. Results in the second week after theray

\begin{tabular}{|c|c|c|c|c|}
\hline & & Group I $(n=41)$ & Group II(n=37) & $\mathrm{P}$ \\
\hline Absence of umbilical exudate & $\mathrm{n}(\%)$ & $35(\% 94.5)$ & $37(\% 100)$ & 0.49 \\
\hline $\begin{array}{l}\text { Presence of umbilical exudat } \\
\text { Complication }\end{array}$ & $\mathrm{n}(\%)$ & $2(\% 5.4)$ & - & 0.49 \\
\hline Burn & $\mathrm{n}(\%)$ & & - & \\
\hline $\begin{array}{l}\text { Skin discoloration } \\
0.49\end{array}$ & $\mathrm{n}(\%)$ & $2(\% 5.4)$ & - & \\
\hline
\end{tabular}

\section{Discussion}

It has not been reported that spontaneously be resolved of UG, which is a commonly seen pathological entity at pediatric surgery outpatient clinics (Hossain et al., 2010; Saleh 2016). It has been reported that it may lead to serious and life-threatening complications, such as omphalitis, sepsis or necrotizing fasciitis when it is left untreated (Fiaz et al., 2017). In populations with limited access to medical care 
and resource poor setting, in order to prevent mortality and morbidity, it is very important to be treated with a reliable, easily accessible and cheap agent by families at home

It was reported by Schmitt in 1972 that table salt could be used for the treatment of umbilical granuloma. The mechanism of action of the table salt is suggested to be its desiccant effect and other biologic properties. When the table salt is applied topically, it causes water to be absorbed out of the granuloma cells without harming the surrounding tissues. This leads to the shrinkage and eventual necrosis of the granuloma (Hossain et al., 2010; Saleh 2016). Some studies reported that recovery rate of 55\%-80\% applying table salt twice a day for a period of three days (Annapurna et al., 2015; Fiaz et al., 2017) while other studies reported a $96 \%-100 \%$ recovery rate after treatment with table salt twice a day for a period of five days (Badebarin et al., 2018; Saleh 2016; Marzban 2008). Hossain et al. conducted a study on 48 infants, applying table salt twice a day for a period of three weeks and reported a recovery rate of $91.7 \%$. However, they added that there was a misdiagnosis in the patients in whom no recoveries were observed, therefore, they argued that the recovery rate was actually 100\% (Hossain et al., 2010). No complications or side effects were reported in none of these studies after the treatment with table salt. Farhat et al.reported that the patients recovered in a shorter period of time without complications treatment with table salt treatment for 24- hours (Farhat et al., 2008). In our study, table salt was applied twice daily for three days for 80 of $\%$ patients and $20 \%$ of patients for six days. İn this study it was observed that all of the patients recovered without any complications or side effects. In our study results may be suggested that as the duration of treatment with table salt is prolonged, the rate of recovery is improved.

The most popular treatment modalities for the treatment of UG is topical silver nitrate (Dhungel et al., 2008; Fiaz et al., 2017; Nagar 2001; Ogowa 2018; Sheth et al., 1990). Silver nitrate has antiseptic, shrinking, and caustic features depending on the concentration and duration of application. Several disadvantages of this treatment modality have been reported including the requirement for treatment by health care personnel at hospitals, the need to apply the treatment more than once, and complications such as the development of discolorations, burns or necrosis in the skin unless care is exercised (Daniel 2001; Majiga et al., 2005). In our study, $23.3 \%$ of the first group were needed to apply silver nitrate twice. Our study results demonstrate that as the number of courses of treatment with silver nitrate increases, the recovery rates also improve however, the complication rates increase too. Farhat et al. study investigated the efficacy of table salt and silver nitrate in the treatment of UG. They reported that table salt was more effective, was cheaper, and more easily available in the treatment compared to silver nitrate (Farhat et al., 2008). Dhungel et al. reported that the recovery rate was $95 \%$ and $4 \%$ reversible minor complication including slight bluish discoloration of skin after the table salt application. After the treatment silver nitrate, the authors reported that the recovery rate was $91 \%$ and they observed $18.8 \%$ complication including minor burns and ulcerations in the skin and $7 \%$ recurrence. They found that the treatment costs were higher in silver nitrate treatment group compared to that of the table salt treatment group (Dhungel et al., 2008).

In conclusion, table salt is a safe alternative to silver nitrate cautery in the treatment of UG. For many populations with limited access to medical care, table salt may be the first choice in the treatment of UG because of the easy access, the ability to treat themselves safely at home, and in-expensive form of treatment.

All of the authors declare that they have all participated in the design, execution, and analysis of the paper, and that they have approved the final version. Additionally, there are no conflicts of interest in connection with this paper, and the material described is not underpublication or consideration for publication elsewhere.

\section{References}

Annapurna D., Ramu P., 2015. Therapeutic Effect of Copper Sulphate vs Common Salt (Table/Cooking Salt) on Umbilical Granuloma in Infants: A Comparative Study. Journal of Evolution of Medical and Dental Sciences. 4:1616-21. doi :10.14260/jemds/2015/228.

Aydın M., Orman A., Deveci U., Taskin E., 2015. Topical clobetasol propionate may not be safe for treating umbilical granuloma in infants. Acta Paediatr. 104:e49-50. doi: 10.1111/apa.12849.

Badebarin D., Abadi S.A., Shakeri M.M , Jamshidi M., Shayan FK., 2018. Comparison between the Clinical Results of Salt Therapy and Surgery in the Treatment of Umbilical Granuloma in Infants. Iranian Journal of Pediatric Surgery. 4(1):7-13 DOI:htt//dx.doi.org/10.22037/irjps.v3i3.20374. 
Brodsgaard A., Nielsen T., Molgaard U., 2015. Treating infants for umbilical granuloma with topical clobetasol propionate cream.Acta Paediatr. 104:e49-50 doi: 10.1111/apa.12824.

Dhungel S., Pokhrel A., Acharya A., 2018. Outcomes and Cost Analysis of Salt Therapy Versus Silver Nitrate for Treatment of Umbilical Granuloma : 4-years Experience in a Private Health Centre .ECRONICON Paediatrics. 7.7:653-659.

Daniel J., 2001. Is silver nitrate the best agent for management of umbilical granulomas? Arch Dis Child. 85:432.

Fiaz M., 2017. A Comparative Study of the Therapeutic Effects of Copper Sulphate versus Common Salt ( Sodium Chloride) in the Treatment of Infantile Umbilical Granuloma. Journal of Medical Science and Clinical Research. 11:31127-31132.doi.org/10.18535/jmscr/v5i11.226.

Farhat A.S., Mohammadzadeh A., 2008. Comparison between two and twenty -four hours salt powder in treatment of umbilical granuloma. Iranian Red Crescent Medical Journal. 10:267-9.

Hossain AKM.Z., Hasan G.Z., Islam KM.D., 2010. Therapeutic Effect of Common Salt (Table/Cooking Salt) on Umbilical Granuloma in Infants. Bangladesh J Child Health. 34:99-102 doi:10.3329/bjch. V34i3.10360.

Lotan G., Klın B., Efratı Y., 2002. Double-ligature: A treatment for pedunculated umbilical granulomas in children. Am Fam Physician. 65:2067-8.

Marzban A., 2008. Curative Effect of Common Salt on Umbilical Granuloma. Journal of Medical Council of Islamic Republic of Iran. 26:373-7.

Majiga V.S., Kumaresan P., Glass E.J., 2005. Silver nitrate burns following umbilical granuloma treatment. Arch Dis Child.2005;90 (7);674. DOI:10.1136/adc.2004.067918.

Nagar. H., 2001. Umbilical granuloma: a new approach to an old problem. Pediatr Surg Int (2001) 17:513-514. DOI: $10.1007 / \mathrm{s} 003830100584$.

Ogawa C., Sato Y., Suzuki C., Mano A., Tashiro A., Niwa T., 'et al'” 2018. . Treatment with silver nitrate versus topical steroid treatment for umbilical granuloma: A non-inferiority randomized control trial. PLoS One 2018;13(2): e0192688. Doi:10.1371/journal.pone.0192688.

Pomeranz A., 2004. Anomalies, abnormalities and care of the umbilicus. Pediatr Clin North AM 2004;51:819. DOI: 10.1016/j.pcl.2004.01.010.

Saleh A.A., 2016. Therapeutic effect of common salt on umbilical granuloma in infants. International Journal of Medical Science and Public Health 2016;5(5):9114.doi:10.5455/ijmsph.2016.07012016312.

Sheth S.S., Malpani A., 1990. The management of umbilical granulomas with cryocautery. Am J Dis Child 1990;144-146. DOI: 10.1001/archpedi.1990.02150260024017. 